\title{
Atuação de acadêmicos de enfermagem nas ações de enfrentamento da covid-19 em comunidades ribeirinhas do amazonas: relato de experiência
}

\author{
Performance of nursing academics in covid-19 facing steels in amazon ribeirin \\ communities: experience report
}

Desempeño académico de enfermería en aceros frente a covid-19 en comunidades ribeirin amazonas: informe de experiencia

José Carlos Ferreira Pinheiro Junior ${ }^{1}$, Gabriel Maia Batista ${ }^{1}$, Jean Lacier Ramos Caxeixa ${ }^{1}$, Mariana Paula da Silva ${ }^{1}$, Joeverson Ferreira Chota ${ }^{1}$, Mayana Cris Duarte Paz ${ }^{1}$, Ramanda Sena Guimarães ${ }^{1}$, Victor Linec Maciel Barbosa ${ }^{1}$, Marcelo Henrique da Silva Reis ${ }^{1 *}$, Jéssica Karoline Alves Portugal ${ }^{1}$.

\section{RESUMO}

Objetivo: Relatar a vivência dos estudantes do curso de Enfermagem no combate a covid-19 nas comunidades ribeirinhas de um município do estado do Amazonas. Relato de experiência: Trata-se de um relato de experiência do tipo descritivo, obtido através do acompanhamento dos discentes do $10^{\circ}$ período do curso de Enfermagem que, integraram uma equipe multiprofissional na realização de ações do enfrentamento a covid-19, na zona rural de um município no interior do Amazonas, no período de julho a agosto de 2020. Estas ações fizeram parte da programação de testagem em massa da população urbana e rural do município, realizada através de sua Secretaria Municipal de Saúde, uma vez que, os ribeirinhos também merecem atenção, por serem vulneráveis e não terem contato frequente com veículos de comunicação que os informem do atual cenário mundial. Considerações finais: As ações de testagem do Covid-19 nesta população, possibilitou o tratamento imediato dos casos positivos e consequentemente a redução no número de óbitos. A atuação dos estudantes foi de suma importância para o amadurecimento de habilidades e técnicas, enquanto futuros profissionais da área da saúde, contribuindo assim com o município neste momento em que a união de esforços é preciso para reduzir a disseminação do vírus.

Palavras-chave: Infecções por coronavírus, População rural, Enfermagem.

\begin{abstract}
Objective: To report the experience of students of the Nursing course in combating covid-19 in the riverside communities of a municipality in the state of Amazonas. Experience report: This is a descriptive experience report, obtained through the monitoring of students from the 10th period of the Nursing course, who were part of a multidisciplinary team in carrying out actions to confront the covid-19, in the rural zone of a municipality in the interior of Amazonas, from July to August 2020. These actions were part of the mass testing schedule for the urban and rural population of the municipality, carried out through its Municipal Health Department, since the riverside residents also they deserve attention, as they are vulnerable and do not have frequent contact with communication vehicles that inform them of the current world scenario. Final considerations: The testing actions of Covid-19 in this population, enabled the immediate treatment of positive cases and consequently the reduction in the number of deaths. The performance of the students was of paramount importance for the maturation of skills and techniques, as future health professionals, thus contributing to the municipality at a time when joint efforts are needed to reduce the spread of the virus.
\end{abstract}

Keywords: Coronavirus infections, Rural population, Nursing.

1 Universidade Federal do Amazonas (UFAM), Coari - AM. *E-mail: reis.henrique.marcelo@gmail.com 


\section{RESUMEN}

Objetivo: Informar la experiencia de los estudiantes del curso de Enfermería en el combate a la covid-19 en las comunidades ribereñas de un municipio del estado de Amazonas. Informe de experiencia: Se trata de un relato de experiencia descriptivo, obtenido a través del seguimiento de estudiantes del décimo período de la carrera de Enfermería, quienes formaron parte de un equipo multidisciplinario en la realización de acciones de enfrentamiento al covid-19, en la zona rural de un municipio del interior de Amazonas, de julio a agosto de 2020. Estas acciones formaron parte del cronograma de pruebas masivas para la población urbana y rural del municipio, realizadas a través de su Secretaría Municipal de Salud, ya que los pobladores ribereños también merecen atención, ya que son vulnerables y no tienen contacto frecuente con vehículos de comunicación que les informen del escenario mundial actual. Consideraciones finales: Las acciones de prueba de Covid-19 en esta población, permitieron el tratamiento inmediato de los casos positivos y, en consecuencia, la reducción del número de muertes. El desempeño de los estudiantes fue de suma importancia para la maduración de habilidades y técnicas, como futuros profesionales de la salud, contribuyendo así al municipio en un momento en el que se necesitan esfuerzos conjuntos para reducir la propagación del virus.

Palabras clave: Infecciones por coronavirus, Población rural, Enfermería.

\section{INTRODUÇÃO}

A covid-19, doença causada pelo novo coronavírus, identificada pela primeira vez em dezembro de 2019 na cidade de Wuhan, China, figura a mais recente ameaça à saúde global. A doença já se espalhou por todo globo, afetando populações de todas as classes sociais, raças e etnias, causando impactos incalculáveis na economia e na vida das pessoas em todo o mundo (WHO, 2020).

No Brasil, o primeiro caso da doença foi confirmado na cidade de São Paulo, no dia 26 de fevereiro de 2020, o paciente era um senhor de 61 anos, com histórico de viagem para a Itália (BRASIL, 2020). No amazonas, o primeiro caso confirmou-se no dia 13 de março de 2020, uma mulher de 39 anos que havia chegado recente de uma viagem feita para Londres (BRASIL, 2020). Diante deste cenário, o Governo Estadual começou a estabelecer medidas restritivas para prevenção da infecção da doença e sua propagação, apesar disto, em abril, um mês após o surgimento do primeiro caso, o Estado já registrava mais de 1.000 casos e 62 mortes (PORTUGAL JKA, et al., 2020).

Os povos ribeirinhos vivem isolados, em áreas que não contam com sistema de saneamento básico. Á água é proveniente do rio, ou em alguns casos de poços artesianos, consumida em grande parte sem um tratamento específico. Em geral o fornecimento de energia só existe para as comunidades mais próximas da zona urbana. A maioria das casas são construídas em madeira e a economia gira em torno da caça, pesca e dos produtos agrícolas cultivados em suas comunidades que são vendidos para micro empresários ou em feiras na sede urbana (FRANCO EC, et al., 2015).

Esses indivíduos residem em regiões geográficas de difícil acesso, rios, lagos, igarapés e seguem a dinâmica dos rios, nos meses de dezembro a julho ocorrem a cheia, favorecendo a locomoção, porém é o período em que não ocorrem a produção de produtos agrícolas. Nos meses de julho a novembro os rios secam, possibilitando a produção dos produtos agrícolas, mas, a locomoção fica comprometida. A canoa movida a motor, é o principal meio de transporte, outras embarcações maiores também são utilizadas para o transporte de passageiros e mercadorias. Sabe-se que, não há assistência de saúde contínua nestas comunidades. (BARTOLI E, 2018; GAMA ASM, et al.,2018).

A pandemia da Covid-19 nos revela o cenário da saúde em comunidades ribeirinhas, estando ligada ao modo de vida no território em que vivem, habitada por pessoas em condições de vulnerabilidade social, vivendo em situação de extrema pobreza, onde muitas políticas públicas não chegam. Mesmo encontrado casos leves da doença, as equipes da atenção primária em saúde, encontram problemas estruturais de acesso a água, produtos de higiene, segurança alimentar e saneamento básico, condições desfavoráveis, que favorecem a disseminação do vírus (CODEÇO CT, et al., 2020). 
Nessas comunidades, a atuação de profissionais de saúde é feita esporadicamente, visto que não há unidades de saúde nessas localidades. Em regiões mais distantes, a oferta de serviços de saúde é mais demorada, porém, a assistência a esses povos melhora em municípios contemplados com a Unidade Básica de Saúde Fluvial, que é utilizada especificamente em municípios da Amazônia legal e pantanal sulmatogrossense, que com o apoio dessa grande ferramenta podem alcançar as pessoas residentes nos locais mais distantes do território nacional (REIS MHS, et al., 2020).

As Unidades Básicas de Saúde Fluviais (UBSF), contam com equipe formada por multiprofissionais da área de saúde. Essa modalidade de cobertura é preconizada pelo Ministério da Saúde através da Política Nacional de Atenção Básica (PNAB), por meio da portaria MS/GM no 2.488 e 2.490, ambas de 2011, desta forma, possibilitando avanços na oferta de saúde da população ribeirinha, destacando a universalidade de acesso, sendo este um dos princípios do Sistema Único de Saúde (SUS) (BRASIL,2012).

As comunidades ribeirinhas, apesar de serem consideradas isoladas, estão ligadas as zonas urbanas, pois seus residentes necessitam se deslocar para venderem seus produtos, buscar serviços públicos de saúde, dentre outras necessidades. Este fluxo constante acarreta riscos para o contágio da covid-19, e consequentemente a disseminação do vírus para a comunidade em que vivem (ANDRADE LC, et al., 2020).

A Secretaria de Estado do Meio Ambiente do Amazonas (SEMA), elaborou um manual ilustrado, adaptado a linguagem local, denominado "fica na comunidade". Tendo como objetivo orientar a população ribeirinha sobre as formas de prevenção da covid-19 e reduzir os impactos causados pela doença nessas localidades. A cartilha reúne informações seguindo as orientações da Organização Mundial da Saúde (OMS) (BRASIL, 2020).

Diante do exposto, o objetivo deste estudo é relatar a vivência dos estudantes do curso de Enfermagem no combate a covid-19 nas comunidades ribeirinhas de um município do estado do Amazonas, além disto, descrever a realidade em que vivem esses povos.

\section{RELATO DE EXPERIÊNCIA}

Trata-se de um relato de experiência do tipo descritivo, obtido através do acompanhamento dos discentes do $10^{\circ}$ período do curso de Enfermagem que, integraram uma equipe multiprofissional na realização de ações do enfrentamento a covid-19, na zona rural de um município no interior do Amazonas, no período de julho a agosto de 2020. A participação dos discentes está assegurada por meio da portaria Portaria $n^{\circ} \mathbf{3 5 6}$, de 20 de março de 2020, do Ministério da Educação (MEC), que autoriza a participação dos estudantes do último ano da graduação de Enfermagem (MEC, 2020).

Além dos discentes de Enfermagem, a equipe era formada por multiprofissionais, sendo eles, médicos, enfermeiros, técnicos de enfermagem, fonoaudiólogo, nutricionista. O município conta com 119 agentes comunitários de saúde (ACS) que residem nas comunidades ribeirinhas, e durante as ações deram todo suporte a equipe no decorrer das atividades.

Estas ações fizeram parte da programação de testagem em massa da população urbana e rural do município, realizada através de sua Secretaria Municipal de Saúde (SEMSA). Uma vez que, os ribeirinhos também merecem atenção, por serem vulneráveis e não terem contato frequente com veículos de comunicação que os informem do atual cenário mundial.

Os mesmos se deslocam até o município, para receberem seus incentivos governamentais, além de venderem seus produtos agrícolas e procurarem serviços públicos de saúde, entre outros. Configurando assim, risco em contrair o vírus durante a estadia na cidade e consequentemente levar o vírus para as comunidades.

As viagens aconteciam em pequenas embarcações, lanchas equipadas com motor potência $150 \mathrm{HP}$ (Horse Power), com capacidade para 12 pessoas, para facilitar o acesso as comunidades que apresentem maior dificuldade de entrada devido as épocas de vazante dos rios e lagos, além disto, trata-se de um deslocamento mais rápido. Na saída da sede do município, eram levados alimentos, água, suprimentos e insumos médicos para serem realizadas as consultas e a realização do teste rápido para detectar a covid-19. 
Ao chegar na comunidade, a equipe organizava o ponto de atendimento aos ribeirinhos em espaços comunitários ou em escolas presentes nos interiores. Os pacientes passavam por uma triagem para prosseguirem ao atendimento médico e posterior avaliação. Em seguida era realizada a notificação dos casos suspeitos, e a realização do teste rápido.

Nos casos positivos os pacientes recebiam a medicação completa seguindo os protocolos clínicos para o tratamento da covid-19, além de álcool em gel e máscaras, para a proteção dos demais moradores que residiam com a pessoa infectada. Uma peculiaridade desta população refere-se ao núcleo familiar ampliado, convívio comunitário, que facilita a disseminação do vírus. Em concomitância ao covid-19, o estado enfrentava o período sazonal de outras síndromes gripais como a H1N1, N3N2. Diante disso, os ribeirinhos foram imunizados contra a influenza, conforme preconizado pelo Ministério da Saúde.

Realizava-se educação em saúde explicando o que era a doença, formas de contágio e prevenção, dentre elas, a lavagem das mãos e uso de máscaras, além da importância do distanciamento social. Ocorreram visitas domiciliares para pacientes sintomáticos da doença que estavam impossibilitados fisicamente de ir até o local de atendimento. Os ribeirinhos eram cercados por inúmeras dúvidas, pelo fato de ser uma doença nova, e também pelo fato de estarem isolados geograficamente e ter a carência de informações veiculadas nas mídias, visto que nem todos possuem acesso aos de meios de comunicação (rádio, televisão e internet).

Vale ressaltar que, frente a situação de pandemia, faz-se necessária a adoção de medidas de prevenção, e a equipe dispunham de todos os Equipamentos de Proteção Individuais (EPIs), preconizados pela OMS, para a segurança dos profissionais envolvidos e da população que recebeu atendimento. $O$ acesso a comunidades localizadas em igapós e igarapés eram dificultadas pela seca, processo que inicia nos meses de julho, e causam impacto na locomoção pela diminuição do nível dos rios. Nesses casos a equipe se deslocava em "voadeiras" embarcação menor que, possibilitou a chegada a essas comunidades.

Foi possível observar que a Covid-19, somou a outras enfermidades que acometem os ribeirinhos, como a malária, presente na região amazônica, problemas gastrointestinais ocasionados por microrganismos patogênicos presentes na veiculação hídrica utilizada para diferentes usos, sem receber algum tipo de tratamento adequado para o consumo e acidentes ocasionados por animais peçonhentos. Pela dificuldade de acesso aos serviços de saúde, os ribeirinhos costumam utilizar seus conhecimentos tradicionais para tratarem estas e outras enfermidades, a partir de chás de plantas medicinais, retirados diretamente da floresta.

A participação enquanto discentes, contribuiu para o desenvolvimento de habilidades e técnicas adquiridas desde os períodos iniciais da graduação. Ainda para o aprimoramento no conhecimento da realidade em que estes moradores vivem, as dificuldades que enfrentam diariamente. Poder contribuir com a saúde local no atual cenário em que vivemos, foi gratificante, pois observamos que é necessária a união de forças para vencermos este vírus invisível, que assola o mundo, neste caso aumentando gradativamente entre as populações ribeirinhas do estado do Amazonas.

\section{DISCUSSÃO}

A atuação de estudantes da área da saúde no enfrentamento a Covid-19 no Brasil, está embasada por meio da Portaria no 356, de 20 de março de 2020, do Ministério da Educação (MEC), que permite os estudantes de enfermagem do último ano da graduação a atuarem no atual contexto de pandemia de Covid19 (MEC, 2020). No âmbito internacional, no Reino Unido, houve o chamamento dos estudantes de enfermagem e medicina dos últimos anos de graduação para somarem forças e participarem de forma remunerada em instituições de saúde (SWIFT A, et al., 2020).

Essas atividades durante a pandemia são consideradas como oportunidades para o desenvolvimento e amadurecimento de competências que vem sendo adquiridas desde os anos iniciais da graduação, além de somar com os profissionais da linha de frente no combate a Covid-19 (MILLER DG, et al., 2020).

É imprescindível a utilização de EPIs pelos profissionais de saúde, principalmente os que atuam diretamente no enfrentamento da doença, pelo grande potencial de transmissão e disseminação do vírus, ao lidarem com casos suspeitos ou confirmados, acarretando em risco de contaminação secundária aos profissionais e uma possível transmissão para outras pessoas de seu convívio social (LAl C, et al., 2020). 
Em decorrência da Covid-19, a campanha de vacinação contra a influenza foi antecipada em todo território nacional, tendo como objetivo auxiliar os profissionais de saúde a descartarem a influenza na triagem de casos para o novo Covid-19, com intuito de facilitar o diagnóstico e minimizar os impactos nos serviços de saúde (BRASIL, 2020).

As comunidades tradicionais da Amazônia, apesar de serem consideradas isoladas pela localização geográfica, estão interligadas diretamente as áreas urbanas através da rede hidrográfica da região, pela necessidade de os ribeirinhos manterem vínculo econômico e acessar os serviços públicos e privados essenciais. A vinda dos ribeirinhos nas cidades no período da pandemia, implica em risco para contraírem a Covid-19 (BARTOLI E, 2018; FREITAS ARR, et al., 2020).

Fatores geográficos e climáticos, como o período de seca na Amazônia, prejudicam o trânsito e transporte fluvial, nos rios, lagos e igarapés, dificultando a circulação da população, profissionais de saúde e insumos, sendo um empecilho ao acesso aos serviços. $E$ consequentemente isolando geograficamente algumas comunidades (PESSOA VM, et al., 2018; GAMA ASM, et al., 2018). Neste contexto, a carência de recursos para qualquer tratamento técnico-cientifico de saúde e a escassez de saneamento básico, foram observadas nas comunidades visitadas, exigindo que as equipes multiprofissionais realizassem a coordenação do cuidado (BOCHNER R, et al., 2012; SAVASSI LCM, et al., 2018; SARTI TD, 2020).

É preciso haver um direcionamento cada vez mais frequente para a população ribeirinha, na oferta de serviços de saúde e de melhorias na qualidade de vida. A pandemia gerou um grande impacto na economia desta população, por realizarem a comercialização de seus produtos nas zonas urbanas, ocorrendo elevadas reduções nas vendas, ocasionado pelo distanciamento social. As ações de testagem do Covid-19 nesta população, possibilitou o tratamento imediato dos casos positivos e consequentemente a redução no número de óbitos, além de possibilitar um panorama situacional nessas localidades. A atuação dos estudantes foi de suma importância para o amadurecimento de suas habilidades e técnicas, enquanto futuros profissionais da área da saúde, contribuindo assim, com o município neste momento em que a união de esforços é preciso para reduzir a disseminação do vírus.

\section{REFERÊNCIAS}

1. ANDRADE LC, et al. Os potenciais impactos da pandemia do COVID-19 nas comunidades ribeirinhas da Amazônia Central e as soluções recomendadas para mitigação. Nota técnica COVID-19 no 004 30/04/2020. Manaus, AM; 2020.

2. BARTOLI E. Cidades na Amazônia, sistemas territoriais e a rede urbana. Mercator; 17: 1-16. 2018.

3. BOCHNER R, et al. Problems associated with the use of medicinal plants commercialized in "Mercadão de Madureira", Rio de Janeiro City, Brazil. Brazilian Journal of Medicinal Plants.14(3):537-47. 2012.

4. BRASIL. Ministério da Saúde. Brasil confirma primeiro caso da doença. Coronavírus. Brasília: Ministério da Saúde, 2020.

5. BRASIL. Ministério da Saúde. Idosos serão os primeiros a receber vacina contra gripe. Coronavírus (Covid-19). Brasília: Ministério da Saúde, 2020.

6. BRASIL. Ministério da Saúde. Política Nacional de Atenção Básica. Brasília: Ministério da Saúde, 2012.

7. BRASIL Secretaria de Estado de Saúde do Amazonas. Amazonas confirma 1ำ caso de Covid-19. Coronavírus. Manaus: Secretaria de Estado de saúde do Amazonas. Disponível em: http://www.saude.am.gov.br/visualizarnoticia.php?id=4327. Acesso em: 18 ago. 2020.

8. BRASIL. Secretaria de Meio Ambiente. GUIA DE CUIDADOS NAS UNIDADES DE CONSERVAÇÃO AÇÕES CONTRA O CORONAVÍRUS (COVID-19). Manaus: Secretaria de Meio Ambiente do Estado do Amazonas, 2020.

9. CODEÇO CT, et al. Risco de espalhamento da COVID-19 em populações indígenas: considerações preliminares sobre vulnerabilidade geográfica e socioeconômica. Relatório $\mathrm{n}^{0}$ 4. $2^{\underline{a}} \mathrm{Ed}, 2020$.

10. FRANCO EC, et al. Promoção da saúde da população ribeirinha da região amazônica: relato de experiência. Rev. CEFAC; 17(5):1521-1530. 2015.

11. FREITAS ARR, et al. Análise da gravidade da pandemia de Covid-19. Epidemiol. Serv. Saude, Brasília, 29(2):e2020119. 2020.

12. GAMA ASM, et al. Inquérito de saúde em comunidades ribeirinhas do Amazonas, Brasil. Cad. Saúde Pública [online]. 34(2): e00002817. 2018.

13. LAI C, et al. Severe acute respiratory syndrome coronavirus 2 (SARS-CoV-2) and coronavirus disease-2019 (COVID19): the epidemic and the challenges. Int J Antimicrob Agents. 55(3):105924. 2020.

14. MILLER DG, et al. The role of medical students during the COVID-19 pandemic. Ann Intern Med. 2020. Disponível em: https://doi.org/10.7326/M20-1281. 
15. Ministério da Educação (BR). Portaria n. 356, de 11 de março de 2020. Dispõe sobre a regulamentação e operacionalização do disposto na Lei no 13.979, de 6 de fevereiro de 2020, que estabelece as medidas para enfrentamento da emergência de saúde pública de importância internacional decorrente do coronavírus (COVID-19). Diário Oficial da União, 12 mar 2020.

16. PESSOA VM, et al. Como garantir o direito à saúde para as populações do campo, da floresta e das águas no Brasil? Saúde Debate; 42(1):302-14. 2018.

17. PORTUGAL JKA, et al. Percepção do impacto emocional da equipe de enfermagem diante da pandemia de COVID19: relato de experiência. Revista Eletrônica Acervo Saúde, n. 46, p. e3794-e3794, 2020.

18. REIS MHS, et al. O impacto do advento de uma Unidade Básica de Saúde Fluvial na assistência aos povos ribeirinhos do Amazonas. Revista Eletrônica Acervo Saúde, n. 53, p. e3631, 9 jul. 2020.

19. SARTI TD, et al. Qual o papel da atenção primária à saúde diante da pandemia provocada pela COVID-19? Epidemiol. Serv. Saúde, Brasília, 29(2):e2020166, 2020.

20. SAVASSI LCM, et al. Saúde no caminho da roça. Rio de Janeiro: Editora Fiocruz; 2018.

21. SWIFT A, et al. COVID-19 and student nurses: a view from England. J Clin Nurs. 2020.

22. WHO. Doença por coronavírus 2019 (COVID-19): relatório de situação - 36. Genebra: Organização Mundial da Saúde. Disponivel em :https://www.who.int/docs/default-source/coronaviruse/situation-reports/20200225-sitrep -36-covid19.pdf? Sfvrsn = 2791b4e0_2. abre em uma nova guia. Acesso em 14 ago.2020. 